\title{
Metamorphosis of Acute to Chronic Pain: A Novel Era of Discoveries in the Field of Pain Medicine
}

\section{Bilal F Shanti ${ }^{1 *}$, Zaynab I Shanti ${ }^{2}$ and Ihsan F Shanti ${ }^{3}$}

${ }^{1}$ Founder and Medical Director of Omnia Pain Consultants and Anesthesiologist with Formal US Fellowship Training in Pain Medicine and Practicing Pain Medicine in a Private Pain Clinic in Phoenix, AZ, USA

${ }^{2}$ Second Year Medical Student at the Royal College of Surgeons Ireland, The

University of Medicine and Health Sciences in Dublin, Ireland

${ }^{3}$ Founder and Medical Director of The American Clinic for Interventional Pain and Spine and Anesthesiologist with Formal US Fellowship Training in Pain Medicine and Practicing Pain Medicine in a Private Pain Clinic in Amman, Jordan

*Corresponding Author: Bilal F Shanti, Founder and Medical Director of Omnia Pain Consultants and Anesthesiologist with Formal US Fellowship Training in Pain Medicine and Practicing Pain Medicine in a Private Pain Clinic in Phoenix, AZ, USA.
Received: September 16, 2021

Published: November 10, 2021

(C) All rights are reserved by Bilal F Shanti., et al.

\begin{abstract}
Chronic pain poses a tremendous burden on economies worldwide. It is among the top causes of work absenteeism. It costs the world hundreds of billions in losses. Pain physicians and other healthcare clinicians face major challenges in treating pain experienced by individuals with persistent pain. How acute pain becomes chronic offers so many theories but little solutions. It is a complex syndrome as complex as pain pathophysiology. It encompasses complex sensory, immune inflammatory, and endocrine interactions at the levels of cerebrum, spinal cord, and peripheral levels. When repeated and/or continuous nerve stimulation precipitates a series of altered pain pathways, resulting in central sensitization and impaired central nervous system mechanisms. The discovery of the roles of PKMzeta, BDNF, and NGF is among the most important in the field of pain medicine. It is a matter of time that PKMzeta inhibitors, BDNF and NGF modulators will lead us closer to discovering novel therapeutic agents that will block acute pain from becoming chronic. The goal of this manuscript is to review the latest and most interesting developments in this filed.
\end{abstract}

Keywords: Metamorphosis; Chronic Pain; Pain Medicine; PKMzeta; BDNF; NGF

\section{Introduction}

Pain is a national public health challenge. According to a report issued by the Institute of Medicine of the National Academies, it is estimated that pain costs the US up to $\$ 635$ billion per year and affects about 100 million American adults-more than the total affected by heart disease, cancer, and diabetes combined [1].
This article will elucidate the latest and possibly the most interesting developments in the field of pain medicine. In Preparing this article, the authors noticed that the published articles ae either too old or too recent, emphasizing a renewal of interest in pain memory and transition of acute pain to chronic. 
Pain clinicians often come across simple pain experiences that linger for a long time for no known reasons. We are running out of affordable pharmacological agents that have limited side effects. The recent wave of opioid-related deaths, addiction, and opioid misuse has prompted many researchers to look beyond traditional pharmacological agents. Pain clinicians must appreciate pain researchers for elevating this field from theory to practice.

Review of the chronic pain theories

An article published this year, out of King's College, sought to get some answers. Researchers examined immune cells in the central nervous system of mice. They found that nerve damage can change epigenetic marks on some of the genes in these immune cells. These cells still behaved normal but carried epigenetic marks that may mean they carry a 'memory' of the initial injury possibly affecting proteins that may determine pain chronicity and recurrence [2].

Another important publication out of the University of Texas at Dallas found that Dopamine may play a role in promoting chronic pain persistence. This chemical that is typically associated with cognition, movement, and reward-motivation maybe a culprit in promoting chronic pain, a title that both norepinephrine and serotonin couldn't hold in previous studies. Researchers found that by removing a collection of neurons called A11 that contain dopamine, chronic pain was selectively diminished, and a toxin that destroyed A11 in mice even permanently reversed a chronic pain state while preserving the acute pain signals [3].

These findings may explain many clinical observations in several pain syndromes in humans. For example, researchers from the University of Michigan found out that one in six new adult patients who reported having chronic pain in childhood are more likely to experience pain of greater severity that is neuropathic in nature and meets clinical criteria for a diagnosis of fibromyalgia [4].

The role of Microglia cells and chronic pain has been under the radar for about two decades. But a study out of Rutgers University is the first to pinpoint the exact role of these cells in the initiation and maintenance of chronic pain. Wu and colleagues theorized that chronic neuropathic pain caused by nerve damage because of an injury could be greatly reduced in animals if the injury was treated targeting microglial cells within the few initial days after the injury.
In other words, depleting the microglia before the nerve injury takes place can permanently prevent it [5].

A 10-year long longitudinal brain imaging study conducted by Apkarian, and his team tried to explain why patients either go on to recover or develop chronic pain. The team concluded that the more 'emotionally' the brain reacts to the initial injury, the more likely the pain will persist after the injury has healed. Ironically, the team could predict with $85 \%$ accuracy at the beginning of the study, which participants would go on to develop chronic pain based on the level of brain imaging interaction between the frontal cortex and nucleus accumbens. Chronic pain participants lost gray matter density, which was likely linked to fewer synaptic connections or neuronal glial shrinkage. These synapses are essential for communication between neurons [6].

A study out of Japan published in 2015, demonstrated that chronic pain stimuli and intense pain have effects at a cellular and/or gene expression level, and will eventually induce "cellular memory due to pain", which means that tissue damage, even if only transient, can elicit epigenetically abnormal transcription/translation and post-translational modification in related cells depending on the degree or kind of injury or associated conditions. Such cell memory/transformation due to pain can cause an abnormality in a fundamental intracellular response, such as a change in the three-dimensional structure of DNA, transcription, or translation. It emphasized on the role of nucleus accumbens playing a role in predicting the value of a noxious stimulus and its offset, and in the consequent changes in the motivational state [7].

Research out of Mexico tried to explain the shaping of synaptic plasticity. It described how $\mathrm{Ca}\left({ }^{2+}\right)$ entry through N-methyl-daspartate-type glutamate neurotransmitter receptors result in the activation of specific signaling pathways leading to changes in gene expression. This concluded that different epigenetic mechanisms (histone acetylation, methylation, and phosphorylation DNA methylation, and hydroxymethylation) underlie learning and memory [8].

Sun., et al. went a bit further by exploring the role of Electroacupuncture. They re-visited the role of pain memory which is related to the phosphorylation of cAMP response element-binding protein (p-CREB) in anterior cingulate cortex. In rats, they found results 
suggesting that retrieval of pain memory could be alleviated by pre-treating with Electroacupuncture, which they attributed at least partially, to the down-regulated expression and combined capacity of $\mathrm{p}$-CREB and the decreased expression of p-CREB in astrocytes and microglia cells [9].

An interesting study out of Seattle Washington, by Han., et al. in 2015, found that a learned 'threat memory', a learned pain behavior to avoid further harmful painful stimulus in mammals, require the amygdala. It tried to explain this mechanism. It found that neurons expressing calcitonin gene-related peptide (CGRP) in the parabrachial nucleus are critical for relaying pain signals to the central nucleus of amygdala and that this pathway may transduce the affective motivational aspects of pain. Genetic silencing of CGRP neurons blocks pain responses and memory formation, whereas their optogenetic stimulation produces defensive responses and a threat memory. They theorized that the identification of the neural circuit conveying affective pain signals may be pertinent for treating pain conditions with psychiatric comorbidities [10].

A study published in 2012 out of The Brain and Mind Research Institute, University of Sydney, demonstrated that pathological neuropathic pain is characterized by microglial activation that is unwanted and considered to contribute to or even cause tactile allodynia, hyperalgesia, and spontaneous pain. Such abnormal microglial behavior seems likely due to an ill-understood disturbance of microglial functions unrelated to inflammation [11]. This theory introduced the new concept of microglial cells and microglial pain.

Another study, also published in 2012, documented the same finding. The traditional microglial function of phagocytosis in CNS was revisited. Persistent activation of the microglial population may contribute to continued dysfunction including chronic pain. It presented evidence for the role of persistent microglial activation in chronic pain after peripheral and central nervous system injury, as well as in diabetic pain, post-herpetic neuralgia pain and related diseases. This emphasized the roles of microglial cells in chronic pain which will hopefully lead to targeted treatments to attenuate or alleviate chronic neuropathic pain syndromes [12]. In addition to microglial cells, another study published in 2016 by Peng., et al. added the role of monocytes to microglia in synergistically promoting the transition from acute to chronic pain after a nerve injury [13].
"Game changing" novel theories on acute pain metamorphosing to chronic pain

The central nervous system is endowed with one "gifted" advantage: It's ability to adapt. This adaptation is called plasticity. Recent advances in molecular neurobiology of pain led to better understanding how neural circuitry maintain long-lasting plasticity leading to chronic pain [14].

"Game changing" research by Ling., et al. [15] demonstrated that spinal dorsal horn neurons might carry an engram representing a molecular mechanism of central sensitization that may promote pain. The maintenance of late synaptic Long-Term Potentiation (L-LTP) necessitates an atypical protein kinase $\mathrm{C}$ called protein kinase PKMzeta. Similar work was reiterated by Sacktor., et al. [16] in 2008. PKMzeta is a unique gene product from the PKCzeta gene. It lacks a regulatory region and is thus autonomously active at the synapse after translation [16]. PKCzeta not only maintains L-LTP but also long-term memory [17].

In 2006, Pastalkova., et al. demonstrated that PKMzeta was sufficient to induce LTP and inhibiting PKMzeta during L-LTP causes LTP to decay [18]. Sacktor., et al. displayed the crucial role of PKMzeta in initiating and maintaining L-LTP linking it to long-term memory trace [16].

Melemedjian and his team went further and monumentally demonstrated that it may well be that other PKCs (kinases) might have a role in the initiation of chronic nociceptive sensitization, but they do not take part in maintaining a chronic pain state. He postulated that a ZIP-reversible process is responsible for the maintenance of persistent sensitization [19]. He added the role of brain derived neurotrophic factor (BDNF) in playing a major role in initiating and maintaining persistent nociceptive sensitization through a ZIP-reversible phenomenon [19]. He theorized that BDNF not only regulates the control of PKMzeta by enhancing its phosphorylation but also has control effect on other PKCs [19]. Melemedjian concluded that BDNF, through its receptor tyrosine receptor kinase type B (trkB) acts as a key regulator in PKC synthesis and phosphorylation and plays a vital role in maintenance of persistent and chronic pain [19]. This landmark finding is the first to directly demonstrate that neurotransmitter-neurotrophin relationship is related to initiation and maintenance of central chronic pain state [19]. 
Obata and Noguchi emphasized the role of BDNF. Its synthesis take place in the dorsal root ganglion (DRG). It is then released into the spinal dorsal horn, and binds to its receptors, trkB. BDNF acts as a neuromodulator of synaptic transmission and spinal nociception. It was found that exogenous BDNF increases NMDA receptorinduced depolarizations in the spinal cord contributing to central sensitization. BDNF mRNA and protein expression is also significantly altered in the DRG after peripheral inflammation and nerve injury. All of this emphasizes the role of BDNF in nociceptive pathways for chronic pain [20].

Recently, it was postulated that nerve growth factor (NGF), through its receptor trkA or p75, may have a role in regulation of PKMzeta [21]. Zhang., et al. opined that NGF appears to regulate dorsal root ganglion excitability via a PKMzeta-dependent process [21]. His thorough research demonstrated that NGF increased the excitability of small-diameter capsaicin-sensitive sensory neurons via a PI3K-mediated, and possibly a ceramide-dependent activation/synthesis of the atypical PKC, PKMzeta [21].

\section{Conclusion}

These theories are closer to clinical realities. Moving these findings from experimental to plausible clinical helpful therapeutic options is closer than ever. Studies on causes of sustenance of plasticity, central sensitization, and chronic pain are amassing massive research work. The discovery of roles of PKMzeta, BDNF, and NGF is a game changer. PKMzeta inhibitor, BDNF and NGF modulators are taking us closer to discovery of novel therapeutic agents that will interrupt the metamorphosis of acute pain into catastrophic chronic sensitization, chronic pain syndromes, and plasticity.

\section{Future Directions}

This particular area of pain is truly 'restless'. The traditional thinking that only a few areas or elements in the brain are responsible for, or are involved in pain, pain initiation, simple transition from acute to chronic pain, and pain memory is soon expiring. It is rapidly becoming evident that brain has multiple central areas involved in pain physiology and pathology, neurobiology, molecular biology, and pain persistence.

The recent introduction of PKMzeta and BDNF concept into the neuro-molecular biology of pain, has been a game changer. Shifting theories from experimental to clinical studies will hopefully con- tribute to finding multiple and novel pharmacological treatments to target the early stages of pain before it gets too late.

\section{Bibliography}

1. Relieving Pan in America. "A Blueprint for Transforming Prevention, Care, Education, and Research. Report Brief June 2011". Institute of Medicine of the National Academies (2011).

2. Franziska Denk., et al. "Persistent Alterations in Microglial Enhancers in a Model of Chronic Pain". Cell Reports (2016).

3. JY V Kim., et al. "Spinal Dopaminergic Projections Control the Transition to Pathological Pain Plasticity via a D1ID5-Mediated Mechanism". Journal of Neuroscience 35.16 (2015): 6307.

4. American Pain Society. "Links of childhood pan to adult chronic pain, fibromyalgia”. Science Daily (2013).

5. Targeting brain cells to alleviate neuropathic pain: Scientists say research indicates that chronic condition could be prevented". Science Daily (2016).

6. MN Baliki., et al. "Corticostriatal functional connectivity predicts transition to chronic back pain". Nature Neuroscience (2012).

7. M Narita., et al. "Pain and Emotional Dysregulation: Cellular Memory Due to Pain". Nihon Shinkei Seishin Yakurigaku Zasshi 35.4 (2015): 89-95.

8. J Cortés-Mendoza J., et al. "Shaping synaptic plasticity: the role of activity-mediated epigenetic regulation on gene transcription". International Journal of Developmental Neuroscience 31.6 (2013): 359-269.

9. J Sun., et al. "Electroacupuncture alleviates retrieval of pain memory and its effect on phosphorylation of cAMP response element-binding protein in anterior cingulate cortex in rats". Behavioral and Brain Functions 11 (2015): 9.

10. S Han., et al. "Elucidating an Affective Pain Circuit that Creates a Threat Memory”. Cell 162.2 (2015): 363-374.

11. MB Graeber and MJ Christie. "Multiple mechanisms of microglia: a gatekeeper's contribution to pain states". Experimental Neurology 234.2 (2012): 255-261.

12. Hulsebosch CE. "Special issue on microglia and chronic pain". Experimental Neurology 234.2 (2012): 253-254. 
13. J Peng., et al. "Microglia and monocytes synergistically promote the transition from acute to chronic pain after nerve injury". Nature Communications 7 (2016): 12029.

14. Woolf CJ. "Central sensitization: Implications for the diagnosis and treatment of pain". Pain 152.3 (2010): S2-S15.

15. Ling DS., et al. "Protein kinase Mzeta is necessary and sufficient for LTP maintenance". Nature Neuroscience 5 (2002): 295-296.

16. Sacktor TC. "PKMzeta, LTP maintenance, and the dynamic molecular biology of memory storage". Progress in Brain Research 169 (2008): 27-40.

17. Sacktor TC. "How does PKMzeta maintain long-term memory?" Nature Reviews Neuroscience 12.1 (2011): 9-15.

18. Pastalkova E., et al. "Storage of spatial information by the maintenance mechanism of LTP”. Science 313 (2006): 1141-1144.

19. Melemedjian OK., et al. "BDNF regulates atypical PKC at spinal synapses to initiate and maintain a centralized chronic pain state". Molecular Pain 9 (2013): 12.

20. Obata K and Noguchi K. "BDNF in sensory neurons and chronic pain”. Neuroscience Research 55.1 (2006).

21. Zhang YH., et al. "Nerve growth factor enhances the excitability of rat sensory neurons through activation of the atypical protein kinase C isoform, PKMzeta". Journal of Neurophysiology 107.1 (2012): 315-335.

Volume 4 Issue 12 December 2021

(C) All rights are reserved by Bilal F Shanti., et al. 